\title{
Juramentos y promesas de profesionales de las áreas que trabajan con animales. Fundamentación para una promesa bioética de los científicos que experimentan con animales (Parte I $)^{1}$
}

Oaths and promises of professionals in areas that work with animals. Groundwork for a bioethical promise of scientists who experiment with animals (Part I)

Recibido: 09 de marzo de 2015 - Revisado: 30 de diciembre de 2015 - Aceptado: 12 de enero de 2016

\section{Luis Fernando Garcés Giraldo ${ }^{2}$}

\section{Resumen}

En este artículo se hace una revisión de algunos juramentos y promesas de profesionales de la salud tanto de animales humanos como de animales no humanos. Se reflexiona sobre el interés que se tiene en que los profesionales realicen un juramento ético al momento de su graduación y sobre la existencia de otros juramentos. Se describe y analiza el Juramento hipocrático, su historia y las variaciones que ha tenido en el tiempo. Por último se identifican los juramentos existentes para los profesionales de las áreas que trabajan con animales en países como Chile, México, Venezuela, Perú y el actual juramento de los médicos veterinarios, los médicos veterinarios zootecnistas y los zootecnistas de acuerdo con el artículo 9 de la ley 576 del 2000 de Colombia.

\section{Palabras clave}

Promesa bioética, virtud, ley 576 del 2000 de Colombia, juramento, Aristóteles.

\begin{abstract}
This article reviews some oaths and promises of health professionals of both human animals and non-human animals. It reflects on the interest people have for the professionals taking an ethical oath upon graduation and on the existence of other oaths. It describes and analyzes the Hippocratic Oath, its history and the changes it has had over time. Finally it identifies existing oaths for professionals in the areas who work with animals in countries like Chile, Mexico, Venezuela, Peru and the current oath of zootechnician veterinary doctors and zootechnicians in accordance with article 9 of law 576 of 2000 of Colombia.
\end{abstract}

\section{Keywords}

Bioethical promise, virtue, law 576 of 2000 of Colombia, oath, Aristotle.
1 Artículo de reflexión derivado de la investigación realizada en la tesis "Bioética en la experimentación con animales a partir de la ética de Aristóteles. Una reflexión filosófica para el cuidado de lo otro" presentada en la Pontificia Universidad Bolivariana, Medellín, Colombia.

${ }^{2}$ Doctor en filosofía de la Pontificia Universidad Bolivariana y vicerrector de Investigación en la Corporación Universitaria Lasallista, Caldas, Antioquia, Colombia. Correo electrónico: lugarces@lasallista.edu.co

Para citar este artículo use: Garcés, L. (2016). Juramentos y promesas de profesionales de las áreas que trabajan con animales. Fundamentación para una promesa bioética de los científicos que experimentan con animales (Parte I). Revista Civilizar Ciencias Sociales y Humanas, 16(30), 261-272. 


\section{Introducción}

Antes de iniciar con la propuesta sobre la redefinición de la promesa para los profesionales de las áreas que trabajan con animales ${ }^{1}$, es conveniente dar una mirada al juramento en que se basan los médicos para la salud de los humanos: este es el Juramento hipocrático. Revisado el juramento que aparece en la ley 576 del 2000 (artículo 9) para los médicos veterinarios, los médicos veterinarios y zootecnistas y los zootecnistas, es necesario realizar algunas modificaciones al juramento; para ello, se revisan algunos de los que se utilizan en diversos países para diferentes profesiones, otros para los profesionales de las áreas que trabajan con animales y el propio juramento usado en Colombia para los profesionales de las ciencias de los animales.

Para los científicos, los juramentos eticos han cobrado interés en los últimos tiempos como una manera de lograr que los investigadores se comprometan a ser responsables y éticos en las áreas de su desempeño profesional, dentro de una sociedad que tiene que contar con valores humanos, sociales y ambientales, hoy en día tan cuestionados. Según Sana (1991)

[...] algunos ejemplos del creciente compromiso de la comunidad científica en el área de la ética científica fueron el Código de ética para científicos de Uppsala (1984); el Juramento de biólogos del Instituto Tecnológico de Massachusetts, MIT (1987); el Juramento hipocrático para científicos (Nuclear Age Peace Foundation, 1987); el Juramento de Buenos Aires (1988); la Resolución de Toronto (1991); y el Juramento de los científicos para la no-participación en investigaciones con fines militares (citado en Have, 2010, p. 20).

Hay alrededor de noventa iniciativas en el mundo sobre juramentos o compromisos éticos para científicos, varias de las propuestas sugieren que se empleen los juramentos en las ceremonias de graduación. Algunos de estos juramentos son: Juramento hipocrático (IV a.
C.), Oración de Maimónides (1200), Juramento hipocrático versión Ginebra (1948), Juramento para los estadísticos (1952), Juramento de los psicólogos (1954), Juramento hipocrático para científicos (1968), Juramento para científicos naturales (1970), Juramento del ingeniero (1973), Juramento de Buenos Aires (1988) y Juramento de Arquímedes (1990), entre otros; (Lemarchand, 2010).

De manera muy general, la definición de juramento es: un compromiso público que hacen los profesionales y que contiene unos principios para actuar éticamente en la profesión. Have (2010) hablando de lo que puede ser un juramento indica que:

[...] un juramento es un llamado a un principio o a una serie de principios que deben ser universalizables, es decir, que puedan ser aplicados a todos los individuos en circunstancias similares. Así, el juramento es una forma privilegiada de asegurar que las personas se involucren personalmente y se obliguen abiertamente, a través de un compromiso público que se constituye por la declaración de principios éticos que cada uno acepta cumplir (p. 25).

El Juramento hipocrático ha sido en todos los tiempos un documento fundamental para la ética y la deontología médica. Hoy en día es poco común emplearlo en su forma original, aunque sirve de base para otros juramentos y normas similares que defienden las buenas prácticas y éticas médicas (Friedrich, 2013). Algunos de ellos se estudiarán aquí.

\section{El Juramento hipocrático}

El juramento más reconocido en el mundo científico es, sin duda alguna, el Juramento hipocrático ${ }^{2}$, usado en la actualidad por los médicos de la medicina humana, el día de su graduación. Este importante manuscrito que señala unas pautas de la ética médica que deben tener estos profesionales ${ }^{3}$ en el ejercicio de su quehacer profesional, se le atribuye a Hipócrates, 
célebre médico griego. Aunque muchos escritores sostienen que no es de su autoría ${ }^{4}$, sigue siendo para la actualidad un importante código ético que define deberes y responsabilidades de la medicina. Para Lemarchand (2010):

El Juramentohipocrático hasido propuesto como un instrumento para que los científicos se comprometan a utilizar sus conocimientos en beneficio de la humanidad, y para que asuman las pertinentes responsabilidades individuales y colectivas destinadas a evitar daños a la humanidad y a la naturaleza (p. 68).

El Juramento hipocrático se puede tomar como el ejemplo más importante para otras disciplinas en la implementación de códigos de conducta para los científicos en general (Have, 2010, p. 23). Córdoba (2010a) se refiere a la aparición del primer Juramento hipocrático en los años 500 antes de Cristo por Alcmeón de Crotona $^{5}$, médico fisiólogo de Sicilia:

Durante cerca de seis siglos, "contados a partir de la segunda mitad del V a. C.", aparecieron diferentes escritos anónimos, cincuenta y tres en total, que reunidos desde la Antigüedad, conocemos como "Corpus Hippocraticum" y de los cuales los más significativos e influyentes fueron compuestos en los últimos decenios del siglo $\mathrm{V}$ y los primeros del siglo IV a. C., especialmente entre los años 420 y 350 . Es, sin duda, la primera obra magna de la medicina "fisiológica", científica e hipocrática (pp. 112-113).

A continuación se describe el texto original del Juramento hipocrático:

Juro por Apolo médico, por Esculapio, Higía y Panacea, por todos los dioses y todas las diosas, tomándolos como testigos, cumplir fielmente, según mi leal saber y entender, este juramento y compromiso:

Venerar como a mi padre a quien me enseñó este arte, compartir con él mis bienes y asistirles en sus necesidades; considerar a sus hijos como hermanos míos, enseñarles este arte gratuitamente si quieren aprenderlo; comunicar los preceptos vulgares y las enseñanzas secretas y todo lo demás de la doctrina a mis hijos y a los hijos de mis maestros, y a todos los alumnos comprometidos y que han prestado juramento, según costumbre, pero a nadie más.

En cuanto pueda y sepa, usaré las reglas dietéticas en provecho de los enfermos y apartaré de ellos todo daño e injusticia. Jamás daré a nadie medicamento mortal, por mucho que me soliciten, ni tomaré iniciativa alguna de este tipo; tampoco administraré abortivo a mujer alguna. Por el contrario, viviré y practicaré mi arte de forma santa y pura.

No tallaré cálculos sino que dejaré esto a los cirujanos especialistas. En cualquier casa que entre, lo haré para bien de los enfermos, apartándome de toda injusticia voluntaria y de toda corrupción, principalmente de toda relación vergonzosa con mujeres y muchachos, ya sean libres o esclavos.

Todo lo que vea y oiga en el ejercicio de mi profesión, y todo lo que supiere acerca de la vida de alguien, si es cosa que no debe ser divulgada, lo callaré y lo guardaré con secreto inviolable.

Si el juramento cumpliere íntegro, viva yo feliz y recoja los frutos de mi arte y sea honrado por todos los hombres y por la más remota posterioridad. Pero si soy transgresor y perjuro, avéngame lo contrario.

El Juramento hipocrático ha tenido variaciones en el tiempo, hasta llegar a una versión actual del mismo. En 1948 y posteriormente en 1968, la Asociación Médica Mundial en su convención de Ginebra ${ }^{6}$, tradujo al lenguaje del momento los valores éticos expresados en el Juramento hipocrático y los proclamó como promesa del médico al optar al título profesional (Córdoba, 2010b, p. 70). Para este cambio se consideró que los actos inhumanos que habían sido realizados por algunos médicos durante los últimos años desconocían la ética profesional; por esto se planteó en esa Convención una modificación a ese juramento para que estuviera 
en concordancia con las prácticas actuales. El texto es el siguiente:

En el momento de ser admitido como miembro de la profesión médica:

Prometo solemnemente consagrar mi vida al servicio de la humanidad. Otorgar a mis maestros el respeto y la gratitud que merecen.

Ejercer mi profesión a conciencia y dignamente.

Velar ante todo por la salud de mi paciente.

Guardar y respetar los secretos confiados a mí, incluso después del fallecimiento del paciente.

Mantener, por todos los medios a mi alcance, el honor y las nobles tradiciones de la profesión médica.

Considerar como hermanas y hermanos a mis colegas.

No permitiré que consideraciones de edad, enfermedad o incapacidad, credo, origen étnico, sexo, nacionalidad, afiliación política, raza, orientación sexual, clase social o cualquier otro factor se interpongan entre mis deberes y mi paciente.

Velar con el máximo respeto por la vida humana.

No emplear mis conocimientos médicos para contravenir las leyes humanas, incluso bajo amenaza.

Hago estas promesas solemne y libremente, bajo mi palabra de honor.

Si bien en el artículo 2 de la ley 23 de 1981, Colombia adopta el juramento aprobado por la Declaración de Ginebra de la Asociación Médica Mundial, existen algunas diferencias con el texto original de esta declaración; como es la introducción del presupuesto "Enseñar mis conocimientos médicos con estricta sujeción a la verdad científica y a los más puros dictados de la ética" y algunas palabras que se incluyen para apoyar un mejor entendimiento y comprensión de este juramento. El texto del Juramento hipocrático para los médicos humanos en Colombia de acuerdo con la ley 23 de 1981 es el siguiente:

El médico deberá conocer y jurar cumplir con lealtad y honor el siguiente juramento médico:

Prometo solemnemente consagrar mi vida al servicio de la humanidad;

Otorgar a mis maestros el respeto, gratitud y consideración que merecen;

Enseñar mis conocimientos médicos con estricta sujeción a la verdad científica y a los más puros dictados de la ética;

Ejercer mi profesión dignamente y a conciencia;

Velar solícitamente y ante todo por la salud de mi paciente;

Guardar y respetar los secretos a mí confiados;

Mantener incólumes, por todos los medios a mi alcance, el honor y las nobles tradiciones de la profesión médica;

Considerar como hermanos a mis colegas;

Hacer caso omiso de las diferencias de credos políticos y religiosos, de nacionalidad, razas, rangos sociales, evitando que estas se interpongan entre mis servicios profesionales y $\mathrm{mi}$ paciente;

Velar con sumo interés y respeto por la vida humana, desde el momento de la concepción $y$, aun bajo amenaza, no emplear mis conocimientos médicos para contravenir las leyes humanas;

Solemne y espontáneamente, bajo mi palabra de honor, prometo cumplir lo antes dicho. 
Una versión del juramento muy utilizada actualmente, sobre todo en países anglosajones, es la redactada en 1964 por el doctor Louis Lasagna, decano de la Facultad de Medicina de la Universidad de Tufts (Estados Unidos). En la versión del "Juramento Lasagna", como se le conoce, se evidencian conceptos nuevos que antes no habían sido tenidos en cuenta como son la importancia de la calidez humana en el trato del médico para con los pacientes; la humildad que se debe tener para la aceptación de las limitaciones de conocimiento y la posibilidad de consultar con otros colegas sobre aspectos que se ignoran; las trampas que se pueden tener en la medicina por el sobretratamiento y el nihilismo terapéutico, y la importancia de la prevención como parte de la labor médica. El texto, en su traducción al castellano, dice así:

Prometo cumplir, en la medida de mis capacidades y de mi juicio, este pacto.

Respetaré los logros científicos que con tanto esfuerzo han conseguido los médicos sobre cuyos pasos camino, y compartiré gustoso ese conocimiento con aquellos que vengan detrás.

Aplicaré todas las medidas necesarias para el beneficio del enfermo, buscando el equilibrio entre las trampas del sobretratamiento y del nihilismo terapéutico.

Recordaré que la medicina no solo es ciencia, sino también arte, y que la calidez humana, la compasión y la comprensión pueden ser más valiosas que el bisturí del cirujano o el medicamento del químico.

No me avergonzaré de decir "no lo sé", ni dudaré en consultar a mis colegas de profesión cuando sean necesarias las habilidades de otro para la recuperación del paciente.

Respetaré la privacidad de mis pacientes, pues no me confían sus problemas para que yo los desvele. Debo tener especial cuidado en los asuntos sobre la vida y la muerte. $\mathrm{Si}$ tengo la oportunidad de salvar una vida, me sentiré agradecido. Pero es también posible que esté en mi mano asistir a una vida que termina; debo enfrentarme a esta enorme responsabilidad con gran humildad y conciencia de mi propia fragilidad. Por encima de todo, no debo jugar a ser Dios.

Recordaré que no trato una gráfica de fiebre o un crecimiento canceroso, sino a un ser humano enfermo cuya enfermedad puede afectar a su familia y a su estabilidad económica. $\mathrm{Si}$ voy a cuidar de manera adecuada a los enfermos, mi responsabilidad incluye estos problemas relacionados.

Intentaré prevenir la enfermedad siempre que pueda, pues la prevención es preferible a la curación.

Recordaré que soy un miembro de la sociedad con obligaciones especiales hacia mis congéneres, los sanos de cuerpo y mente, así como los enfermos.

Si no violo este juramento, pueda yo disfrutar de la vida y del arte, ser respetado mientras viva y recordado con afecto después. Actúe yo siempre para conservar las mejores tradiciones de mi profesión, y ojalá pueda experimentar la dicha de curar a aquellos que busquen mi ayuda.

En 1986, en la ciudad de San Miguel de Tucumán (Argentina), diferentes científicos se reunieron con el objetivo de proponer a la comunidad científica el Juramento hipocrático para científicos; esta idea surgió por iniciativa de algunos de los miembros del comité organizador del Simposio Internacional sobre los Científicos, la Paz y el Desarrollo. Se tuvieron diversas propuestas y al final se concertó una fórmula que hiciera énfasis en la conciencia individual, en donde los candidatos se comprometieran a dedicar su trabajo solo en beneficio de la humanidad y a favor de la paz, no anteponiendo ni la remuneración, ni el prestigio ni subordinarse ante la voluntad de empleadores o dirigentes políticos, asumiendo la responsabilidad total de sus propias acciones (Lemarchand, 2010, pp. 76-79). 
El texto del documento que tomó el nombre de "Juramento de Buenos Aires" fue proclamado el 15 de abril de 1988 ante la Asamblea del Simposio Internacional sobre los Científicos, la Paz y el Desarrollo. Este juramento fue acogido rápidamente por las organizaciones científicas de diversos países y ha sido traducido al ruso, árabe, chino, sueco y alemán, entre otros idiomas. El Juramento de Buenos Aires ha sido firmado por varios científicos de reconocimiento mundial y algunos de ellos ganadores del Premio Nobel: Linus Pauling (Premio Nobel de Química en 1954 y Premio Nobel de la Paz en 1962); Abdus Salam (Premio Nobel de Física en 1979); Sheldon Lee Glashow (Premio Nobel de Física en 1979); además de Carl Sagan, Mario Bunge y Victor F. Weisskopf en 1980. A continuación se presenta el texto del Juramento de Buenos Aires (05 de abril de 1988):

Teniendo conciencia de que la ciencia, y en especial sus resultados pueden ocasionar perjuicios a la sociedad y al ser humano cuando se encuentran ausentes los controles éticos:

¿Juráis que la investigación científica y tecnológica que desarrollaréis será para beneficio de la humanidad y en favor de la paz, que os comprometéis firmemente a que vuestra capacidad como científicos nunca servirá a fines que lesionan la dignidad humana, guiándoos por vuestras convicciones y creencias personales, asentadas en un auténtico conocimiento de las situaciones que os rodean y de las posibles consecuencias de los resultados que puedan derivarse de vuestra labor, no anteponiendo la remuneración de empleadores o dirigentes políticos?

Si así no lo hiciereis, vuestra conciencia os lo demande.

Otra versión de un juramento moderno es la escrita por Roger J. Bulger, presidente de la Universidad de Texas y expresidente de la Asociación Estadounidense de Facultades de Medicina. Este médico considera que el Juramento hipocrático se mantiene vigente porque en su gran calidad personal refleja los conceptos básicos de devoción por el prójimo y del compromiso de servicio; por eso redactó lo que puede denominarse como el "Moderno juramento hipocrático". Este juramento -al igual que el de la Declaración de Ginebra- es la pieza de mayor reconocimiento en las promesas de responsabilidad que tienen los galenos en la actualidad (Patiño, 2004):

Por lo más elevado de mis creencias prometo a mis pacientes idoneidad, franqueza, compromiso con todo lo que mejor pueda servirles, compasión, discreción absoluta y confidencialidad dentro del margen de la ley.

Trataré a mis pacientes como quisiera que a mí me trataran; obtendré interconsultas o segundas opiniones cuando así lo deseen; los educaré para que participen, si así quieren, en toda decisión importante que a ellos incumba. Y procuraré minimizar su sufrimiento si su enfermedad se volviere intratable, en la convicción de que alcanzar una muerte digna debe ser una meta importante en la vida de cada cual.

Trataré de establecer una relación amistosa con mis pacientes y los aceptaré como tales sin pretender juzgar sus actuaciones, comprendiendo que cualquier sistema de valores es sólido y encomiable, y concediendo a cada individuo la totalidad de su dignidad humana.

Cobraré tan solo los honorarios justos por mis servicios profesionales sin tratar de lucrarme, ni financieramente ni en ninguna otra forma, por aquellos consejos y cuidados que dispense a mis pacientes.

Aconsejaré e infundiré ánimo a mis pacientes en todos sus esfuerzos por mejorar su propia salud. Dentro de mi profesión, pondré todos los medios a mi alcance para mejorar la calidad de la atención médica y para fortalecer la salud pública. En mi calidad de ciudadano trabajaré por la equidad en la salud para todos, sin dejar que otras consideraciones de carácter profesional o general interfieran con mi compromiso de proveer la mejor y más adecuada atención a cada uno de los seres bajo mi cuidado. En cuanto pueda vivir en concordancia con los anteriores preceptos, seré un médico realmente valioso (p. 29). 


\section{Algunos juramentos existentes en América Latina para los profesionales de las ciencias que trabajan con animales}

En Chile existe un Juramento hipocrático de los médicos veterinarios escrito por la médica veterinaria Marta Rivas Riveros (profesora de microbiología de la Universidad de Chile) (Friedrich, 2013, p. 35), que fue adoptado por el Colegio Médico Veterinario de Chile. Este juramento se inspira en el Juramento hipocrático original, además de tener algunos elementos propios de la profesión del médico veterinario: la protección de la vida de los animales y evitar su sufrimiento; salvaguardar al hombre de los riesgos de las enfermedades que los animales le puedan transmitir y el uso de los animales como alimento para el hombre. El texto es el siguiente:

Juro que cumpliré con honradez y seriedad todas las finalidades de mi profesión.

Trataré por cuantos medios científicos estén a mi alcance, de proteger la vida de los animales útiles al hombre, así como evitaré el sufrimiento innecesario de los mismos.

Protegeré la salud del hombre de las enfermedades que los animales le puedan transmitir.

Emplearé las técnicas más apropiadas para obtener de los animales los alimentos que beneficien al hombre, que mejoren su nutrición dentro de un amplio servicio social y mejoramiento colectivo.

Respetaré y honraré a mis maestros, estimaré a mis colegas y los trataré conforme a las normas de la ética profesional, e igual cosa haré con las personas que soliciten mis servicios dentro de la medicina de los animales y la zootecnia.

Ofrezco estudiar y superarme permanentemente para poder cumplir con eficacia la labor profesional que tengo encomendada.
El Código de ética profesional del médico veterinario y zootecnista de México, emitido el 17 de agosto de 1999 por la Federación de Colegios y Asociaciones de Médicos Veterinarios Zootecnistas de México (1999), en su artículo 72 declara el juramento que deben realizar los médicos veterinarios zootecnistas en el momento de su graduación:

Consciente de la obligación que acepto como profesional, en este momento solemne, juro que cumpliré los objetivos de la carrera de médico veterinario zootecnista.

Me esforzaré por incrementar al máximo posible la producción de alimentos de origen animal para provecho de la humanidad, por salvaguardar la salud del hombre evitando las enfermedades que los animales puedan transmitirle, y por evitar el sufrimiento innecesario de estos.

Juro que trataré a mis compañeros y a quienes soliciten mis servicios, apegándome estrictamente a las normas de respeto y ética profesional, y que, sin limitación alguna, transmitiré mis experiencias y conocimientos a los miembros de esta profesión y a los aspirantes a realizarla.

Prometo que guardaré gratitud a mi facultad y a mis maestros, y ofrezco estudiar y superarme permanentemente para realizar con eficacia la misión que tengo encomendada.

Me conduciré con honradez y esmero en la aplicación de los conocimientos que he adquirido para beneficio de la sociedad, a fin de llevar con honor el grado que ahora recibo de la universidad que me formó.

El juramento para la Federación de Colegios de Medicos Veterinarios de Venezuela, especificado en la Ley de Ejercicio de la Medicina Veterinaria de Venezuela (1968) de manera particular, establece la protección de la salud de los animales en especial los recursos de la ganadería y la avicultura. El texto dice: 
Habiendo sido admitido a la profesión de médico veterinario, juro solemnemente usar mis conocimientos y habilidades para el beneficio de la sociedad, protegiendo la salud animal, impulsando los recursos de la ganadería y la avicultura; aliviando el sufrimiento de los animales y contribuyendo a mejorar la salud pública y el progreso de la ciencia médica.

Juro ejercer mi profesión con conciencia, dignidad y lealtad, manteniendo los principios éticos de la medicina veterinaria y reconociendo la obligación de continuar mejorando mis conocimientos y aptitudes, mientras ejerza esta noble profesión.

Para el caso del Perú, existe el Código deontológico del Colegio Médico Veterinario del año 2006, en este se propone el juramento que deben realizar los médicos veterinarios al momento de su graduación, cuando el decano de la facultad, o quien haga sus veces, mencione algunas consideraciones personales sobre el ejercicio de la profesión y luego se dirija a los nuevos profesionales así:

Cumplidos todos los requisitos y formalidades de ley y habiendo sido admitidos como miembros del Colegio Médico Veterinario del Perú para ejercer la profesión de médico veterinario, deberéis usar vuestros conocimientos y habilidades para el beneficio de la humanidad protegiendo la salud pública y el medioambiente, impulsando la producción pecuaria, aliviando el sufrimiento de los animales y contribuyendo al progreso de las ciencias veterinarias.

Juráis ejercer la medicina veterinaria con conciencia, dignidad y lealtad, manteniendo los principios éticos y deontológicos, reconociendo la obligación de continuar mejorando vuestros conocimientos y aptitudes mientras ejerza esta noble profesión; y juráis cumplir y hacer cumplir las leyes, estatuto, reglamento, código deontológico y demás normas complementarias que rigen los destinos de la medicina veterinaria.

\section{Juramento en Colombia de los profesionales sanitarios que trabajan con animales}

El juramento para los médicos veterinarios, médicos veterinarios zootecnistas y zootecnistas que se encuentra vigente en Colombia es el consagrado en el artículo 9 de la ley 576 del 2000, sobre el "Código de ética para el ejercicio profesional de la medicina veterinaria y zootecnia". El siguiente es el juramento con el que se consagran estos profesionales el día de su graduación:

Juro, en el nombre de Dios, cumplir la Constitución y las leyes de mi patria y todas las obligaciones inherentes a la profesión de medicina de los animales y la zootecnia.

Protegeré al hombre de las enfermedades que los animales puedan transmitir, y emplearé las técnicas necesarias para obtener de los animales los alimentos que lo beneficien, respetando los ecosistemas y evitando riesgos secundarios para la sociedad y su hábitat mediante el uso de insumos y prácticas con tecnologías limpias, defendiendo la vida en todas sus expresiones.

Honraré a mis maestros, hermanaré con mis colegas y enseñaré mis conocimientos dentro de la misión científica con generosidad y honestidad. Prometo estudiar y superarme permanentemente para cumplir con eficiencia la labor profesional encomendada.

Enalteceré mi profesión cumpliendo bien, siempre y en todo momento, las normas y preceptos de la Ley de Ética Profesional.

En los juramentos en estudio no se observa con claridad algunos compromisos que se considera deben estar incluidos en una promesa para los científicos actuales que experimentan con animales: por ejemplo deberes para con el planeta que muestren la relación de interdependencia que debe existir entre los seres vivos (tanto animales humanos como no humanos). Si 
bien el ser humano debe estimarse como un ser preeminente en la naturaleza, como sujeto ético tiene la responsabilidad de velar por el cuidado de aquellos seres que dependen exclusivamente de él, seres vivos que por sí solos no son capaces de tomar decisiones.

Es en el hombre como ser dotado de razón y responsable de sus actos, en donde deben estar todas las decisiones para hacer de los actos de la experimentación, actos llenos de prudencia, continencia, téchne, sabiduría y justicia, virtudes aristotélicas que se deducen como significativas para estar contenidas en una nueva promesa para los profesionales que experimentan con animales.

Se puede revisar en algunos escritos del mismo autor de este artículo, con mayor justificación, donde se desarrollan cada una de estas virtudes, los aspectos más relevantes para ser tenidos en cuenta por un científico, el modelo bioético elaborado para la experimentación con animales y la forma como se debe proceder para la deliberación por los comités de bioética. Se prevé también, un artículo adicional que comprenda el desarrollo y la explicación de la propuesta para la promesa bioética de los científicos de las áreas que trabajan con animales que podrá ser usada en Colombia y en el mundo por ser de carácter universal.

\section{Conclusión}

Se debe reformular para Colombia y para las áreas de las ciencias que trabajan con animales en general, el juramento con que cuentan los médicos veterinarios, los médicos veterinarios zootecnistas y los zootecnistas (ley 576 del 2000). Es imperioso elaborar un juramento o promesa más universal y que comprenda no solo las virtudes que debe tener un especialista en las ciencias que trabajan con animales, sino también aspectos donde se aclaren las relaciones entre los diferentes seres vivos y la responsabilidad que deben tener los seres humanos, como seres racionales, virtuosos y con decisio- nes que hagan de sus actos, acciones que deban ser alabadas y no censuradas. Para el diseño de la promesa o juramento es forzoso contar con elementos de las virtudes aristotélicas, de los principios universales de las tres erres (principios propuestos por Russell \& Burch en 1959 con la finalidad de producirle al animal de experimentación el menor daño y sufrimiento posibles, que son: reducir, remplazar y refinar; para cada uno de estos principios existen unas estrategias o alternativas que el investigador debe tener en cuenta en el momento de presentar una propuesta de investigación) y de las normas que para Colombia o cada país donde sea adoptado, estén vigentes en el momento que los profesionales hagan dicho juramento o promesa.

\section{Notas}

1 Son aquellos profesionales de las ciencias de la salud y la producción animal que estudian el comportamiento, la biología y los usos productivos y reproductivos de los animales; es decir, médicos veterinarios, médicos veterinarios zootecnistas, zootecnistas, biólogos e ingenieros pecuarios.

2 Córdoba (2010a) nos cuenta quién fue Hipócrates de Cos: "Muy poco se conoce con certeza de él: nació en la isla de Cos, cuna de ilustres médicos, en el año 460 a. C. Recibió de su padre la primera formación. Ejerció como médico 'periodeuta' en Tesalia, Tracia, proximidades del Ponto Euxino, la isla de Tasos, y falleció - la fecha es incierta- en Larisa, según algunos alrededor del año 375 a. C., es decir, a los 85 años de edad. Diferentes estudios, especialmente filológicos, sostienen que ninguno de los escritos que forman la colección 'Corpus Hippocraticum' puede atribuirse directamente a la figura más representativa de esta medicina, otros concluyen que solo unos cuantos, entre ellos el 'juramento', son realmente suyos" (pp. 113-114).

3 Para Gracia (2004) profesión es un término de origen religioso. El verbo latino es 
profiteor, profesar. Una persona profesa, es la consagrada a un menester y que lo confiesa públicamente. El profesional es una persona consagrada a un menester, y públicamente reconocida como tal. Mediante el juramento, el neófito adquiere la categoría de profeso, es decir, de profesional. El término profesión tiene aquí un sentido estrictamente religioso, y conlleva por ello el ejercicio de una responsabilidad interna, privada o moral, a la vez que la más estricta impunidad externa, pública o jurídica (p. 133).

${ }^{4}$ Es el caso citado en Lemarchand (2010, p. 68) tal vez uno de los trabajos más profundos, detallados y mejor documentados acerca del origen y significado trascendental del Juramento hipocrático sea el estudio de Ludwig Edelstein (1943). En esta monografía se fundamenta abrumadoramente -casi palabra por palabra- la tesis de que el verdadero origen del juramento se remonta a la Escuela Pitagórica. Para Edelstein el Juramento hipocrático es un documento uniformemente concebido y atravesado profundamente por la filosofía pitagórica. En su espíritu, forma, texto y contenido, el juramento es un verdadero manifiesto pitagórico.

5 Entonces ¿por qué se dice que es el Juramento hipocrático o la medicina hipocrática, si el iniciador de esta modalidad es Alcmeón de Crotona? Al respecto Córdoba (2010a) citando a Laín afirma que "Alcmeón fue el iniciador de la medicina fisiológica; Hipócrates, su verdadero fundador" (p. 113), pues fue ese médico de Cos quien le imprimió con sus enseñanzas y, sobre todo, con el ejemplo de su vida, el carácter del servicio respetuoso, venerativo al ser humano, en tal grado que mereció que pensadores como Platón y Aristóteles reconocieran y elogiaran su labor, que Aristóteles lo llamara 'El más grande', que Galeno lo calificara como 'El divino', 'El inventor de todo bien' y que en general se le conozca como el padre de la medicina.

${ }^{6}$ Para Chavarría (2009) la presente declaración se basa en el Juramento hipocrático. Fue adoptada por la Asamblea General de la
Asociación Médica Mundial en Ginebra, Suiza, en septiembre de 1948, enmendada por la 22a Asamblea Médica Mundial que se celebró en Sídney, Australia, en agosto de 1986; la 35a Asamblea Médica Mundial de Venecia, Italia, en octubre de 1983 y la 46a Asamblea Médica General de la Asamblea Médica Mundial en Estocolmo, Suecia, en septiembre de 1994 y revisada en su redacción por la 170a Sesión del Consejo, Divonne-les-Bains, Francia, en mayo de 2005 y por la 173a Sesión del Consejo, Divonne-les-Bains, Francia, en mayo de 2006 (p. 42).

\section{Referencias}

Chavarría, O. (2009). Declaración de Ginebra. Revista Archivos de Pediatría de Uruguay, 80(1), 42-42. Recuperado de http://www. scielo.edu.uy/scielo.php?pid=S1688$12492009000100009 \&$ script $=$ sci_arttext

Comisión Nacional de Bioética (1993). Declaración mexicana y principios básicos de la experimentación en animales. México: Consejo de Salubridad General.

Córdoba, R. (2010a). La bioética y la práctica médica postmoderna. Medellín: Universidad Pontificia Bolivariana.

Córdoba, R. (2010b). Fundamentación Bioética para el ejercicio de la medicina. Medellín: Universidad Pontificia Bolivariana.

Federación de Colegios y Asociaciones de Médicos Veterinarios y Zootecnistas. (1999). Código de Ética Profesional del Médico Veterinario y Zootecnista de México. México: Federación MVZ.

Friedrich, N. (2013). El Juramento Hipocrático y los veterinarios. Información Veterinaria, 173, 33-35. Recuperado de http:// www.produccion-animal.com.ar/temas varios_veterinaria/139-juramento_hipocratico.pdf 
Gracia, D. (2004). Fundamentación y enseñanza de la bioética. Ética y vida, estudios de bioética 1. Bogotá: El búho.

Have, T. (2010). Hacia un juramento ético universal para científicos. En G. A. Lemarchand (Ed.), Ciencia para la paz y el desarrollo: el caso del Juramento Hipocrático para Científicos (pp. 17-29) Montevideo: UNESCO. Recuperado de http://unesdoc.unesco.org/ images/0018/001884/188400s.pdf

Juramento de Buenos Aires (1988). Juramento hipocrático para científicos. Asamblea del Simposio Internacional sobre los Científicos, la Paz y el Desarrollo, San Miguel de Tucumán, Argentina.

Juramento hipocrático. (IV a. C.). Redactado por Hipócrates (siglo $\mathrm{V}$ a. C).

Juramento hipocrático de los médicos veterinarios. (399 a. C.). Escrito por Marta Rivas y adoptado por el Colegio Médico Veterinario de Chile: Chile.

Juramento Lasagna. (1964). Juramento hipocrático escrito por Louis Lasagna. Estados Unidos: Universidad de Tufts. Recuperado de https://sites.google.com/site/ juramentoshipocraticos/home/versionde-1964

Juramento Moderno. (1988). Moderno Juramento Hipocrático escrito por Roger Bulger. Estados Unidos: Iowa City.

Juramento para la Federación de Colegios de Medicina Veterinaria de Venezuela. (2008). Venezuela.
Lemarchand, G. (Ed.). (2010). Ciencia para la paz y en beneficio de la humanidad: El concepto del Juramento Hipocrático para Científicos. En, Ciencia para la paz y el desarrollo: el caso del Juramento Hipocrático para Científicos (pp. 39-110). Montevideo: UNESCO. Recuperado de https://arxiv.org/ftp/arxiv/papers/1006/1006.3527.pdf

Ley 23 de 1981. Por la cual se dictan normas en materia de ética médica. Diario Oficial No. 35.711 Congreso de la Republica de Colombia, febrero de 1981.

Ley 576 de 2000. Por la cual se expide el Código de Etica para el ejercicio profesional de la medicina veterinaria, la medicina veterinaria y zootecnia y zootecnia. Diario Oficial No 43.897. Congreso de la Republica de Colombia, febrero de 2000.

Patiño, J. (2004). En busca del Hipócrates moderno. Persona y Bioética, 8(23), 22-33.

República del Perú. (2006). Código deontológico del Colegio Médico Veterinario de Perú. Perú. Recuperado de http://www. cmvl.pe/wp-content/uploads/2013/04/ Codigo-Deontologico.pdf

Russell, W. M. S., \& Burch, R. L., (1959). The Principles of Humane Experimental Technique. Londres: Methuen.

República Bolivariana de Venezuela. Ley de ejercicio de la medicina veterinaria de 1968. Gaceta oficial No. 28.737. Congreso de la República de Venezuela, septiembre de 1968. 
